\title{
Improvement of Biological Properties of Natural Hemostatic Agent by Plasma Technology
}

\author{
Wassanai Wattanutchariya ${ }^{1}$, Dheerawan Boonyawan ${ }^{2}$ and Jureeporn Jaifu ${ }^{3}$ \\ ${ }^{1}$ Advanced Technology Research Center (AMTech), Department of Industrial Engineering, Faculty of Engineering, Chiang Mai University, \\ Chiang Mai, Thailand \\ ${ }^{2}$ Plasma and Beam Physics Research Facility (PBP), Department of Physics and Materials Science, Faculty of Science, Chiang Mai \\ University, Chiang Mai, 50200, Thailand \\ ${ }^{3}$ Biomedical Engineering Institute (BMEI), Chiang Mai University, Chiang Mai, 50200, Thailand
}

\begin{abstract}
The aim of this study was to evaluate the effect of non-thermal plasma treatment on the biological properties of a natural hemostatic agent. The results show that plasma treatment can enhance the biodegradability property of the hemostatic agent by increasing the degradation rate of the specimen up to $94.26 \%$ within 7 days. Furthermore, the plasma-treated specimen also exhibited good biocompatibility based on the cell viability test of the fibroblast cells. The cell growth and cell proliferation on this sample were found to be helpful for the wound healing process. With appropriate degradable and biocompatible properties, this modified agent could be beneficial for better control over bleeding during surgery. Research on the physical and mechanical properties are on to develop novel hemostatic products to match the requirements as far as biomedical applications are concerned.
\end{abstract}

\section{Introduction}

Bleeding during a clinical procedure is a matter of concern. Excessive hemorrhage is an important problem in surgery. Control of bleeding and rapid blood clotting are primary management tasks in an operation [1]. Thus, an effective hemostatic agent is an important equipment for this purpose. In the last few years, biomaterials have been employed to fabricate commercial use of natural hemostatic agents such as gelatin, rice starch, and oxidized cellulose, but some of them have not been costeffective or had some limitation for the application [2]. Among others, chitosan (CS) has been successfully developed into a hemostatic agent with immediate blood absorption property. CS is a natural polymer which can be derived from crustacean shell, crab exoskeleton, or squid pen. CS is composed of a linear polysaccharide of $\mathrm{N}$-acetyl and D-glucosamine units. A previous study reported that CS was an effective material to fabricate the sponge hemostatic agent by using the freeze-drying technique. The small pores of CS sponge and its surface roughness are good for increased absorption via swelling, and it also has the property of rapid blood absorption. Additionally, CS hemostatic agents can accelerate the hemostasis mechanism of early blood clotting by the electrostatic force, whereby the positive charge of CS reacts with the negative charge of the red blood cell (RBC) membranes [1,3]. Furthermore, CS has been known to exhibit both physicochemical and biological properties such as antimicrobial activity, biodegradability, biocompatibility, and non-cytotoxicity [4,5]. Nowadays,
CS is widely used to stop bleeding in several applications. Therefore, CS was chosen as the raw material to produce the natural hemostatic agent for this investigation.

Generally, the hemostatic agent for some kinds of surgery of critical organs, such as neurosurgery, abdominal surgery, control capillary, and venous and small artery bleeding, is needed to be left inside the patient's body for a prolonged period of time for bleeding control, cell proliferation induction, and wound healing enhancement $[2,6]$. On the other hand, if the hemostatic agent for this specific application cannot be completely degraded within the human body, it can induce the risk of foreign body reactions, inflammation, and infection [7]. According to a previous study, the hemostatic agent can degrade in the body within a specific period [8]. Thus, biodegradation is an important property of an effective hemostatic agent for clinical use. The trends of biodegradability and biocompatibility are key to the indicated development of biomaterials for biomedical applications.

Currently, non-thermal plasma technology is implemented for surface modification and treatment of heat-sensitive materials. It was possible to employ atmospheric pressure plasma jet (APPJ) to modify the surface of several polymers and biomaterials [9], which included a natural hemostatic agent. According to a previous study, RF-plasma could be used to modify the chemical properties of the CS surface, increase the number of amino groups on the CS surface, change the ionic permeability and increase the hydrophilicity of CS membranes [10,11], improve blood clotting properties, 
and increase the degradation rate [12]. Thus, the purpose of this study was to investigate the effect of non-thermal plasma treatment on the biological properties of a natural specimen, especially the biodegradability and biocompatibility prior to the development into a novel hemostatic agent that is a degradable bleeding control product for surgery and other medical applications.

\section{Methodology}

The preparation of the CS hemostatic agent and the APPJ setup experiment in this study were performed by following the process from a previous study [13].

First, the CS solution was prepared by adding $2 \mathrm{~g}$ of the squid pen to $100 \mathrm{ml}$ of deionized (DI) water; then, $1 \%$ of acetic acid was added and the CS solution was left at room temperature for 3 days [13]. Thereafter, $0.025 \%$ of glutaraldehyde was added to the solution as the crosslinking agent, and then the mixture was stirred until it became homogenous. Afterward, this crosslinked solution was poured into 24 well-plates (of $1 \mathrm{ml}$ each), and then the samples were frozen at $-80^{\circ} \mathrm{C}$ for $24 \mathrm{~h}$, followed by freeze-drying at $-50^{\circ} \mathrm{C}$ for $48 \mathrm{~h}$ to form the hemostatic specimens.

Then, the APPJ was setup, as shown in Figure 1. The plasma conditions were also controlled in accordance with the procedure followed in a previous study [13]. This non-thermal atmospheric pressure plasma jet was driven by $330 \mathrm{kHz}$ of radio frequency (RF) power supply and mixed gas flow rates of argon and oxygen of $4 \mathrm{l} / \mathrm{min}$ and $10 \mathrm{ml} / \mathrm{min}$, respectively. The gap between the quartz capillary and the sample surface was $5 \mathrm{~mm}$, and the input power was set at $10 \mathrm{~W}$, with 30 s treatment time for each specimen.

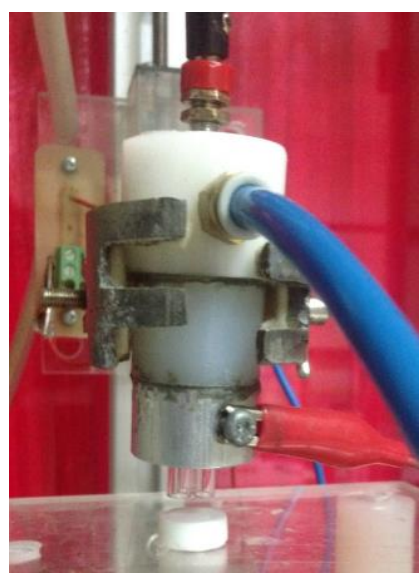

Figure 1. The experimental set up of APPJ for natural hemostatic treatment.

Characterization of the sample was carried out in terms of degradability and biocompatibility to confirm the biological behaviour in conditions mimicking human conditions. The degradation was determined in terms of the weight loss after the samples were immersed in 3 $\mathrm{ml}$ of phosphate buffer saline (PBS) containing lysozyme $(1.6 \mu \mathrm{l} / \mathrm{ml})$, and incubated at $37^{\circ} \mathrm{C}$ [14]. Then, the degraded samples were removed and repeatedly washed with $100 \mathrm{ml}$ DI water for 3 times at predetermined times of 1 day, 3 days, and 7 days. The samples were prefrozen at $-80^{\circ} \mathrm{C}$ for $24 \mathrm{~h}$ in the freezer and then freeze-dried at $-50^{\circ} \mathrm{C}$ for $48 \mathrm{~h}$ [15]. The degradation rate was considered from the final weight of the samples after freeze-drying (Wo) and the initial weight of the samples before they were immersed in PBS with lysozyme (Wt). The percentage of biodegradation was calculated using equation (1):

$$
\text { Degradation }(\%)=\frac{\mathrm{Wt}-\mathrm{Wo}}{\mathrm{Wt}} \times 100
$$

In the meantime, biocompatibility was performed based on the cell viability of the fibroblast cells. In this study, fibroblast cells were cultured until the density of growth reached $1 \times 105$ cells per $\mathrm{cm} 2$ in 24 well-plates, and the samples were left overnight. The cytotoxicity was evaluated with MTT assay. First, the MTT solution $(300 \mu \mathrm{l})$ was added into each well plate, and the plates were incubated at $37^{\circ} \mathrm{C}$ for $4 \mathrm{~h}$ to allow the formation of the formazan crystals; thereafter, the MTT solution was removed, and $1 \mathrm{ml}$ of DMSO was added and the well plate was agitated for $20 \mathrm{~min}$. The values of cell absorbance were measured with a spectrophotometer to be between $540 \mathrm{~nm}$ and $630 \mathrm{~nm}$, and compared between the absorbance value of the cell test with the sample (ABSsample) and the absorbance value of the control (ABScontrol) [4]. The percentage of cell viability was calculated using equation (2):

$$
\text { Cell viability }(\%)=\frac{\mathrm{ABS} \text { sample }}{\mathrm{ABS} \text { control }} \times 100
$$

All the experiments were repeated 5 times and for 2 blocks (10 replicates). The data are expressed as mean \pm SD. The statistical analysis was based on ANOVA, and multiple comparison tests were employed. The difference was considered statistically significant when the $\mathrm{p}$-value was less than 0.05 .

\section{Results and discussion}

Figure 2 shows the photograph of the hemostatic agent during the biodegradation test on day 1 , day 3 , and day 7 , while Figure 3 illustrates the biodegradation rate of these hemostatic agents when compared between the plasma treatment and the non-plasma treatment. As can be seen from the graph, the plasma treatment could significantly improve the biodegradability of the specimen, which is evident by the increase in the percentage of degradation on day 1 from $21.35 \%$ to 
$49.06 \%$, on day 3 from $24.57 \%$ to $66.60 \%$, and, inducing the highest percentage of degradability, on day 7 from $86.48 \%$ to $94.26 \%$. Based on the findings of a previous study [13], it is well-known that APPJ treatment not only enhances the blood absorption ability but also induces higher biodegradation rates of the hemostatic agent.

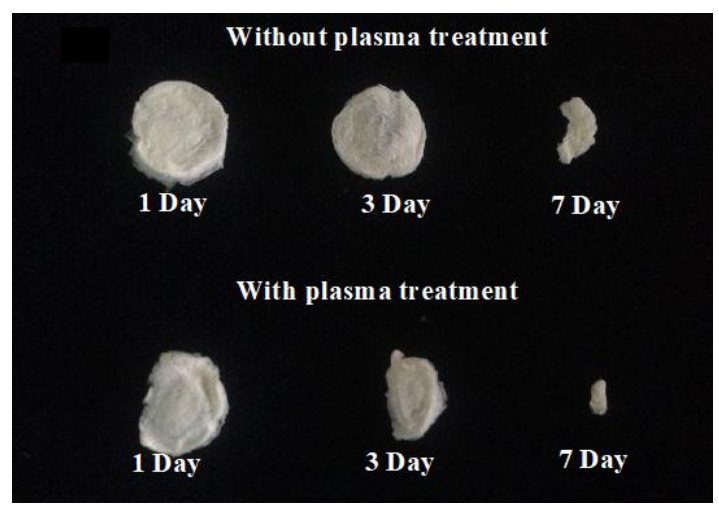

Figure 2. Photograph of the biodegradation of chitosan hemostatic agent.

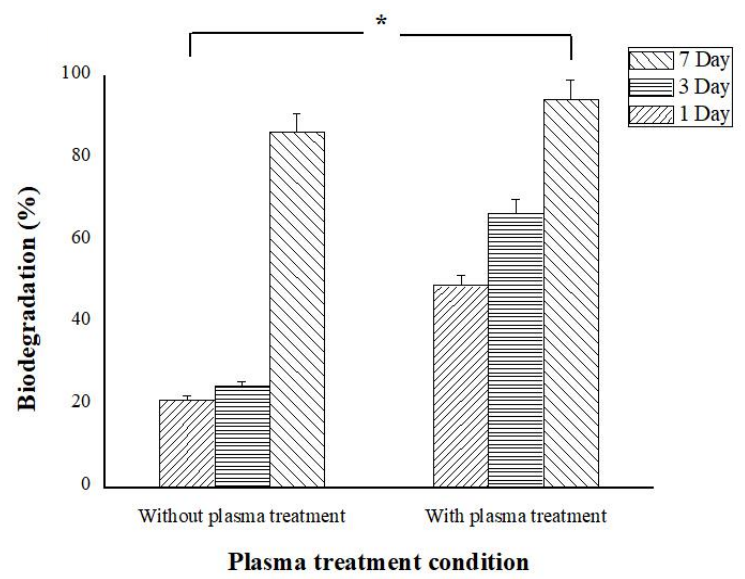

Figure 3. The biodegradation rate of the CS hemostatic agent.

With regard to the setup of APPJ, the $\mathrm{Ar} / \mathrm{O}_{2}$ gas mixture generated the gas discharge of the $\mathrm{OH}$ radical $(308 \mathrm{~nm})$, the argon atom $(696 \mathrm{~nm})$, and the atomic oxygen $(777 \mathrm{~nm})$. Argon has an important role in hydrophilic surface modification through physical etching. The argon atom was affected by the amine group $\left(\mathrm{NH}_{2}\right)$ of $\mathrm{CS}$, which increased the energy of the surface as well as decreased the surface tension, leading to higher surface roughness that helped improve cell adhesion, allow ionic permeability, increase hydrophilicity, enhance blood absorption, and accelerate blood clotting. Even though argon could not create new functional groups, it generated free radicals and polar functional groups on the CS surface, and these free radicals reacted with the oxygen functional groups, which enhanced the hydrophilic properties and induced the fast biodegradability $[11,16]$.

At the same time, the oxygen plasma can discharge an atomic oxygen and an $\mathrm{OH}$ radical; atomic oxygen mechanism is a chemical etching process for surface modification for hydrophilicity. The oxygen activity creates a new polar group from an atomic oxygen $(\mathrm{O}$ 777) and an $\mathrm{OH}$ radical $\left(\mathrm{OH}^{-}{ }^{308}\right)$. The reaction can be classified into two mechanisms, as shown in Figure 4.

Step 1: The first mechanism of atomic oxygen plasma: the $\mathrm{O} \cdot$ breaks the hydrogen bond of the amine $\left(\mathrm{NH}_{2}\right)$ group of $\mathrm{CS}$, and then combines with $\mathrm{O} \cdot$ to form a water molecule $\left(\mathrm{H}_{2} \mathrm{O}\right)$ and nitrogen $\left(\mathrm{N}^{+}\right)$protonated functional group on the CS surface. At the same time, $\mathrm{O} \cdot$ breaks the hydrogen bond of the hydroxyl $(\mathrm{OH})$ group of $\mathrm{CS}$, and then combines with $\mathrm{O} \cdot$ to form a hydrogen bonding $\left(\mathrm{H}^{+}\right)$and species of the functional oxygen group $\left(\mathrm{O}_{2}\right)$. Furthermore, the oxygen gas plasma can discharge the $\mathrm{OH}$ radical; the $\mathrm{OH} \cdot$ breaks the hydrogen bond of the amine $\left(\mathrm{NH}_{2}\right)$ group of $\mathrm{CS}$, and then combines with $\mathrm{OH} \cdot$, leading to the formation of a water molecule $\left(\mathrm{H}_{2} \mathrm{O}\right)$ and functional amine group $\left(\mathrm{NH}^{+}\right)$on the CS surface. Meanwhile, $\mathrm{OH} \cdot$ breaks the hydrogen bond of the hydroxyl $(\mathrm{OH})$ group of $\mathrm{CS}$, and then combines with $\mathrm{OH} \cdot$ to form a water molecule and species of a functional oxygen group $\left(\mathrm{O}^{-}\right)$.

Step 2: The proteases and the lysozyme in the biodegradation test setup help break the glycosidic bonds of the polysaccharide of CS and degrade the particle of CS into oligosaccharides; thereafter, they combine with the metabolic pathway and get excreted [13], resulting in better biodegradation property of the specimen. 
Step 1: $\mathrm{O}_{\bullet}$ and $\mathrm{OH} \cdot$ created new polar group on CS surface.

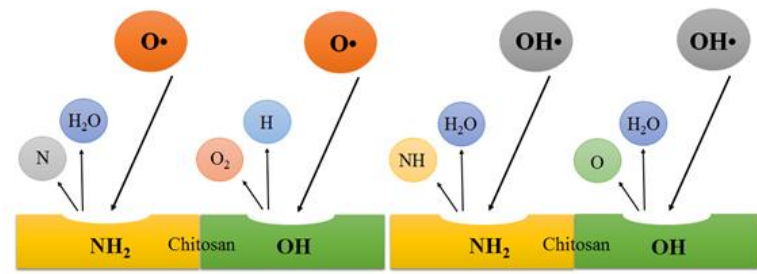

Step 2: $\mathrm{O} \cdot$ and $\mathrm{OH} \cdot$ enhance the biodegradation of the CS surface.

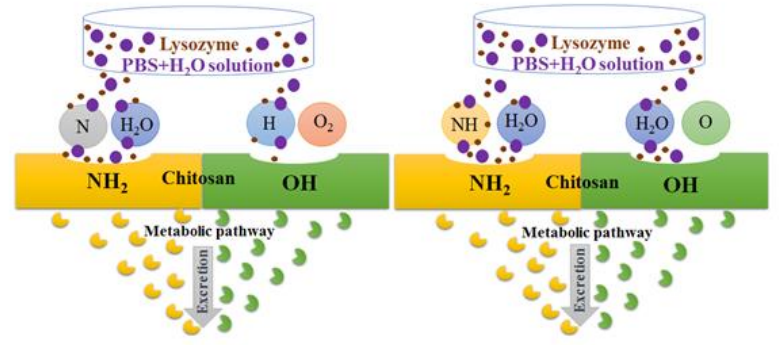

Figure 4. Surface modification of CS hemostatic agent by oxygen plasma.

Finally, the CS hemostatic agents also exhibited high percentage cell viability of fibroblast cells of more than $100 \%$. Figure 5 shows a comparison of the percentages of cell viability of fibroblast cells in the CS hemostatic agent between with and without plasma treatment. The statistical evaluation showed that the biocompatabilty of the hemostatic specimen from the two conditions were not significantly different ( $p$ value $>0.05)$. The percentages of cell viability of the specimen with and without plasma treatment were $111.11 \%$ and $103.43 \%$, respectively. Thus, the plasma treatment was not found to affect cell growth on the CS hemostatic agent.

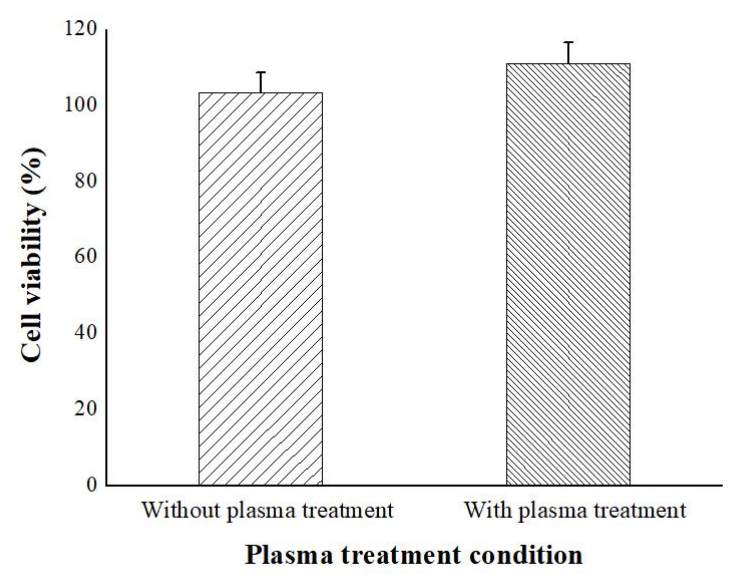

Figure 5. Surface modification of CS hemostatic agent by oxygen plasma.
In fact, CS was biocompatible and non-toxic by itself, so it exhibited a high percentage of cell viability, more than $100 \%$. CS could induce cell growth, support cell adhesion, accelerate the serum plasma and extracellular matrix (ECM) proteins, as well as increase the activation of platelets [17]. In addition, CS treated with $\mathrm{Ar} / \mathrm{O} 2$ gas plasma is also used as a scaffold to absorb the cell-adhesive ability in some molecules such as collagen, fibronectin, vitronectin, and laminin; these molecules interact with the integrins on the Schwann cell (SC) surface to promote cell attachment and cell proliferation [11]. Thus, the present investigation confirms the good biocompatibility, non-toxicity, and ability to induce cell growth, as well as proliferation, of plasma-treated natural hemostatic agents which lead to the enhancement of cell repair and wound healing process, which can be beneficial for better control over bleeding during surgery.

\section{Conclusion}

This study investigated the effect of plasma treatment on the biological properties of a natural hemostatic agent. The results show that the biodegradability of the specimen could be significantly improved. Nonthermal plasma treatment with $\mathrm{Ar} / \mathrm{O} 2$ gas setup enhances the hydrophilic property of the CS specimen due to the excitation of the $\mathrm{OH}$ radicals and the atomic oxygen, leading to surface modification. Furthermore, chitosan was observed to react with the plasma product, which caused higher metabolic pathway and faster excretion of the specimen. Thus, this plasma technique accelerated the degradation rate of the hemostatic agent to be the highest, up to $94.26 \%$, within 7 days. In addition, this plasma-treated hemostatic confirmed its good biocompatibility with fibroblast cells. In vivo studies of the plasma-treated natural hemostatic agent are currently under investigation to confirm the effect of plasma reaction on related properties prior to implementation for surgical use or other related medical applications.

\section{References}

1. Huang X, Sun Y, Nie J, Lu W, Yang L, Zhang Z, Yin $\mathrm{H}$, Wang $\mathrm{H}$ and $\mathrm{Hu} \mathrm{Q}$ J. Biol. Macromol. 75 322-329 (2015)

2. Kumar S, Asian J. Pharm. Clin. Res. 9 (3) 35-41 (2016)

3. Kang PL, Chang SJ, Manousakas I, Chen WL, Yao $\mathrm{CH}$, Lin FH and Kuo SH, J. Curr. Appl. Phys. 11 457-461 (2011) 
4. Tanase CE and Spiridon L, J. Mater. Sci. Eng. C 40 242-247 (2014)

5. Croisier F and Jerome C, J. Eur. Polym. 49 780792 (2013)

6. Glick JB, Kaur RR and Siegel D, J. Indian Dermatol. 4 (3) 172-176 (2013)

7. Emilia M, Luca S, Francesca B, Luca B, Paolo S, Giuseppe F, Gianbattista B, Carmela M, Luigi M and Mauro L, J. Transfus. Apher. Sci. 45 305311(2011)

8. Ding Z, Chen J, Gao S, Chang J, Zhang J and Kang ET, J. Biomater. 25 1059-1067 (2004)

9. Kostov KG, Nishime TMC, Castro AHR, Toth A and Hein LRO, J. Appl. Surf. Sci. 314 367-375 (2014)

10. Uygun A, Kiristi M, Oksuz L, Manolache S and Ulusoy S, J. Carbohydr. Res. 346 259-265 (2011)

11. Huang YC, Huang CC, Huang YY and Chen KS, J. Biomed. Mater. Res. A 10 842-851(2007)

12. Desmet T, Morent R, Geyter ND, Leys C, Schacht E and Dubruel P, J. Bio Macromol. Am. Chem. Soc. 10 (9) 2351-2378 (2009)
13. Jaifu J, Thunsiri K, Udomsom S, Boonyawan D and Wattanutchariya $\mathrm{W}$, J. materials today proceedings (to be published)

14. Frantz VK, J. Ann. surg. 1 116-126 (1943)

15. Yang B, Li XY, Shi S, Kong XY, Guo G, Huang MJ, Luo F, Wei YQ, Zhao X and Qian ZY, J. Carbohydr. Polym. 80 860-865 (2010)

16. Juang RS and Huang C J. Taiwan Inst. Chem. Eng. 54 76-82 (2015)

17. Gomathysankar S, Halim AS and Yaacob NS, J. Arch. Plast. Surg. 41 452-457 (2014) 